\title{
Amélioration de méthodes de modification structurale - Applications
}

\author{
Mathieu Corus ${ }^{2, a}$, Étienne Balmès $^{2}$ et Laurent Billet ${ }^{1}$ \\ 1 EDF DR\&D, 1 Av. du Général de Gaulle, 92141 Clamart Cedex, France \\ 2 École centrale Paris, Grande voie des Vignes, 92295 Chatenay-Malabry, France
}

Reçu le 23 novembre 2004, accepté le 31 mars 2005

\begin{abstract}
Résumé - Une méthode proposant d'estimer l'effet de modifications structurales distribuées est exposée ici. Une évolution des formulations classiques permettant de prendre en compte la non-coïncidence des points de mesure et des points de couplage est présentée. La mise en œuvre de la méthodologie à un exemple académique et à un cas d'application industriel permet de mettre en lumière les avantages de la méthodologie exposée.
\end{abstract}

Mots clés : Modification structurale / analyse modale expérimentale / sous structuration dynamique / modes d'interface / réduction de modèle / expansion de données

\begin{abstract}
Structural dynamic modification methods improvement - Applications. Structural modification methods are designed to estimate the dynamic behaviour of a structure after a modification. This estimation is obtained by coupling an experimental model of the non modified structure and a numerical model of the modification For short delays and little resources, these methods, as presented in [1] for example, can be efficiently implemented. Few authors dealt with the problem of distributed modifications (W. D'Ambrogio and A. Sestieri in [2] and [3], K. Elliot and L. Mitchell in [4], or B. Schwarz and M. Richardson in [5]). Nevertheless, all proposed methods impose a specific test configuration with several measurement points over the substructures interface. The improvements presented here allow the use of data that do not require to be measured on the substructures interface. The difficulties generated by the lack of displacement continuity at the interface as well as the different locations of measurement and coupling points have been overtaken by means of data expansion and model reduction techniques. The expansion process uses a reduced displacement basis for describing the interface. It is derived from a linear combination of the measurement point motions. To select the appropriate basis, two indicators based upon two displacement estimators on the interface are introduced. Two case study are presented and discussed, showing the ability of this approximate approach to accurately foresee structural modification effects.
\end{abstract}

Key words: Structural modification / experimental modal analysis / dynamic sub structuring / interface modes / model reduction / data expansion

\section{Introduction}

Les méthodes de modification structurale permettent d'estimer le comportement dynamique d'une structure après modification en s'appuyant sur un modèle comportemental de la structure non modifiée obtenu à partir d'essais d'une part et d'un modèle numérique de la modification d'autre part. Ces méthodes, présentées dans [1] par exemple, sont particulièrement indiquées pour résoudre les incidents de type vibratoire. Lorsque les contraintes

\footnotetext{
a Auteur correspondant : corus@mssmat.ecp.fr
}

sur les délais et les moyens ne permettent pas de réaliser un modèle numérique représentatif pour évaluer l'effet d'une modification, cette classe de méthodes permet d'exploiter les données d'essais vibratoires.

Peu d'auteurs se sont penchés sur le problème des modifications distribuées (W. D'Ambrogio et A. Sestieri dans [2] et [3], K. Elliot et L. Mitchell dans [4], ou encore B. Schwarz et M. Richardson dans [5]). Cependant, quelle que soit la méthode adoptée par ces auteurs, tous imposent d'avoir des points de mesure sur l'interface pour réaliser le couplage des sous-structures. Telles que décrites dans ces communications, les différents méthodes ne sont 


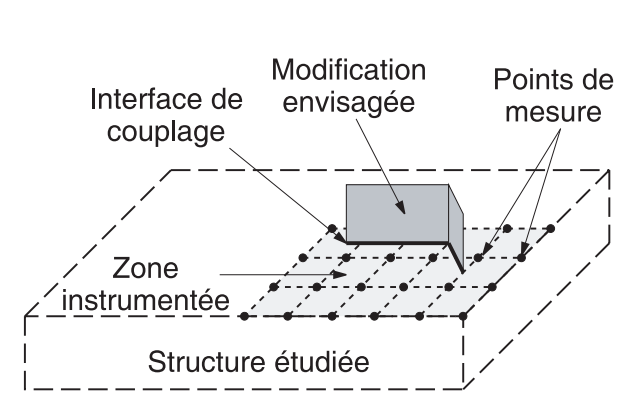

\section{Évolutions permlses}

- Instrumentation fimitée à un sous domaine de la structure étudiée,

- Contínuité de l'interface de couplage,

- Coïncidence interface / points de mesure non nécessaire.

Données requises

- Modèle expérimental de la structure étudiée,

- Modèle numérique (E.F.) de la modification.

Fig. 1. Schématisation des hypothèses adoptées dans le cadre de la méthode présentée.

DÉPlacements $\left\{y_{\mathrm{t}}\right\}$ AUX POINTS DE MESURE

Noeuds du maillage expérimental

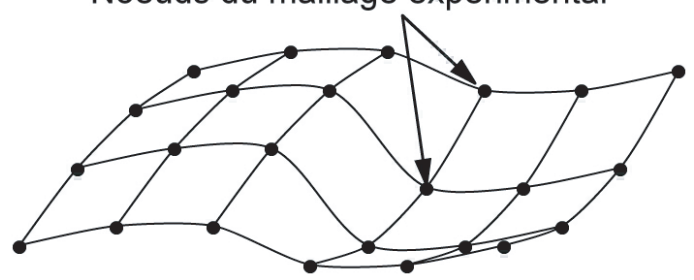

DÉPLACEMENTS $\left\{q_{\mathrm{I}}\right\}$ AUX DDL D'INTERFACE

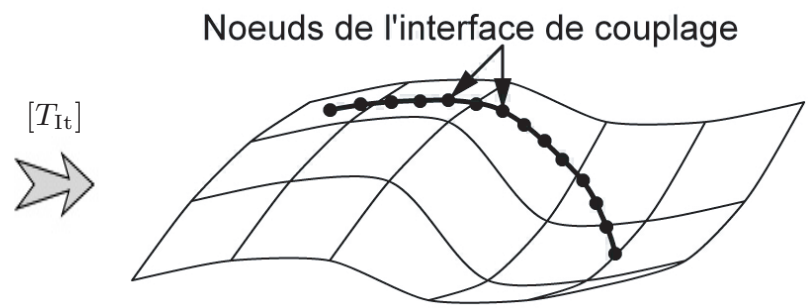

Fig. 2. Reconstruction des mouvements des DDL d'interface à partir des déformées modales identifiées.

donc applicables que dans le cas où l'essai a été réalisé en connaissant au préalable la modification projetée.

L'objectif ici est d'exploiter le plus complètement possible un essai vibratoire quelconque. Un tel essai aura été réalisé pour établir un diagnostic, la modification n'étant proposée qu'après dépouillement de l'essai. On cherche à s'affranchir des deux difficultés que sont la continuité de la modification et la non-coïncidence des points de mesures et des points de couplage. La formulation proposée ici repose sur les travaux réalisés par E. Balmès, notamment dans [6]. L'approche mise en œuvre combine les méthodes d'expansion, de réduction de modèle et de sous-structuration. Après avoir exposé la formulation des évolutions proposées, deux exemples d'applications sont présentés.

\section{Formulation des évolutions proposées}

Cette section résume les principaux points de la méthode proposée. La formulation de l'expansion des données mesurées sur l'interface de couplage est d'abord exposée. Le modèle E.F. retenu pour réaliser l'expansion, appelé modèle local, est présenté. Un indicateur de sélection des vecteurs de la base d'expansion est également proposé.

\subsection{Hypothèses - Principes}

Les évolutions permises et les données requises pour l'application de la méthode présentée sont synthétisées dans la figure 1.

Pour définir le couplage entre le modèle expérimental et le modèle numérique, les déplacements au niveau de l'interface doivent être connus pour les deux sousstructures. Dans le cas général, seuls les mouvements de la modification le sont. L'information manquante pour la structure étudiée doit être estimée. On fait alors une hypothèse forte en supposant que les mouvements de l'interface peuvent être décrits à partir de la seule connaissance des mouvements des points instrumentés. On cherche à construire un opérateur $\left[T_{\mathrm{It}}\right]$ permettant de décrire les mouvements (translations et rotations) des DDL d'interface $\left\{q_{\mathrm{I}}\right\}$ comme combinaison linéaire des déplacements mesurés $\left\{y_{\mathrm{t}}\right\}$. Les mouvements de l'interface correspondant à des déplacements mesurés quelconques peuvent ainsi être estimés. La figure 2 schématise le passage de $\left\{y_{\mathrm{t}}\right\}$ à $\left\{q_{\mathrm{I}}\right\}$.

\subsection{Formulation de l'expansion}

Pour réaliser le couplage, il faut construire un opérateur $\left[T_{\mathrm{It}}\right]$ de sorte qu'on puisse écrire

$$
\left\{q_{\mathrm{I}}\right\} \approx\left[T_{\mathrm{It}}\right]\left\{y_{\mathrm{t}}\right\}
$$

Pour construire $\left[T_{\mathrm{It}}\right]$, il faut se donner une base de déplacement définie sur les points de mesure et sur l'interface de couplage. De nombreuses méthodes existent pour reconstruire des données à partir de mesures discrètes (voir [7] par exemple). La solution retenue ici, détaillée à la section 2.3, consiste à réaliser un modèle E.F. local du sous-domaine instrumenté de la structure étudiée. Les mouvements des DDL d'interface $\left\{q_{\mathrm{I}}\right\}$ et les déplacements aux points de mesure $\left\{y_{\mathrm{t}}\right\}$ sont décomposés sur une base de déplacements $\left[\Phi_{\mathrm{Lg}}\right]$ de ce modèle local, appelés modes d'interface.

Sur la base du formalisme d'entrées/sorties, on construit $\left[C_{\mathrm{tL}}\right]$, la matrice d'observation faisant le lien 


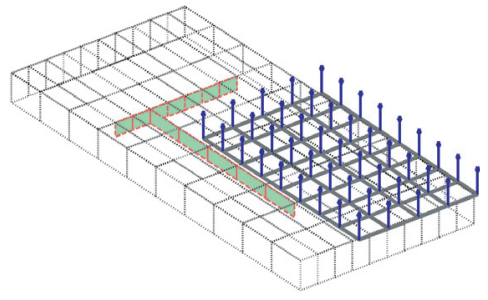

Structure étudiée maillage expérimental

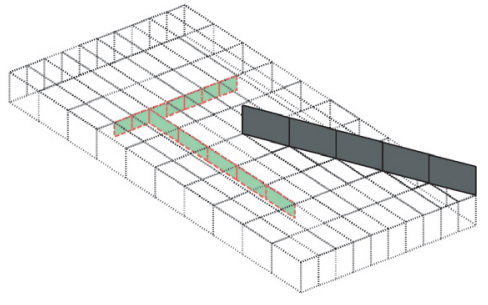

Structure modifiée

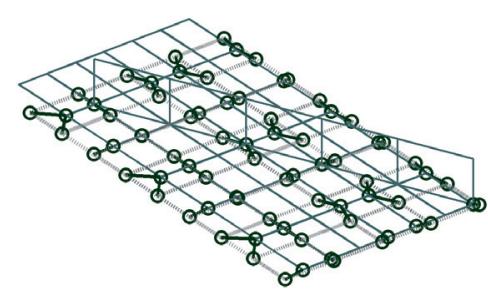

Modèle local - liens avec le maillage expérimental $\left(\left[C_{\mathrm{tL}}\right]\right)$

Fig. 3. Exemple de modèle local et de modification pour une structure donnée.

entre les DDL $\left\{q_{\mathrm{L}}\right\}$ du modèle local et les données de test $\left\{y_{\mathrm{t}}\right\}$. Différentes méthodes de construction de $\left[C_{\mathrm{tL}}\right]$ sont présentées dans [8]. En décomposant ce mouvement sur la base restreinte des déplacements du modèle local, il vient

$$
\left\{y_{\mathrm{t}}\right\} \approx\left[C_{\mathrm{tL}}\right]\left[\Phi_{\mathrm{Lg}}\right]\left\{\eta_{\mathrm{g}}\right\}
$$

On impose que l'interface soit incluse dans les DDL du modèle local. On note $\left[C_{\mathrm{IL}}\right]$ la matrice opérant la sélection de l'interface parmi l'ensemble des DDL. La décomposition des mouvements de l'interface sur $\left[\Phi_{\mathrm{Lg}}\right]$ pour des déplacements mesurés $\left\{y_{\mathrm{t}}\right\}$ s'écrit

$$
\left\{q_{\mathrm{I}}\right\}=\left[C_{\mathrm{IL}}\right]\left[\Phi_{\mathrm{Lg}}\right]\left\{\eta_{\mathrm{g}}\right\}
$$

où $\left\{\eta_{\mathrm{g}}\right\}$ est le vecteur des DDL généralisés associés à la base $\left[\Phi_{\mathrm{Lg}}\right]$.

L'expression de $\left[T_{\mathrm{It}}\right]$ recherchée en (1) est alors

$$
\left[T_{\mathrm{It}}\right]=\left[C_{\mathrm{IL}}\right]\left[\Phi_{\mathrm{Lg}}\right]\left[T_{\mathrm{gt}}\right]
$$

avec $\left[T_{\mathrm{gt}}\right]$ défini par minimisation

$$
\left[T_{\mathrm{gt}}\right]=\operatorname{Arg}\left(\min _{T}\left\|[T]\left\{y_{\mathrm{t}}\right\}-\left\{\eta_{\mathrm{g}}\right\}\right\|^{2}\right)
$$

L'opérateur $\left[T_{\mathrm{It}}\right]$ permet de reconstruire les mouvements de l'interface associés aux modes propres identifiés $\left[\Phi_{\text {test }}\right]$. L'écriture des conditions de continuité des déplacements et de réciprocité des efforts à l'interface pour les deux sous-structures fournit l'équation de la dynamique pour le système couplé. Les modes propres et les valeurs propres du système couplé sont obtenus par la résolution du problème aux valeurs propres obtenu.

Pour que l'équation (5) admette une solution unique, le nombre $N_{\mathrm{g}}$ de vecteurs de $\left[\Phi_{\mathrm{Lg}}\right]$ doit être inférieur ou égal au nombre de capteurs $N_{\mathrm{t}}$. Le résultat de (5) dépendant de $\left[\Phi_{\mathrm{Lg}}\right]$, un indicateur est nécessaire pour déterminer la taille optimale de la base dans la limite de $N_{\mathrm{t}}$ vecteurs.

\subsection{Modèle local - Construction des modes d'interface}

L'estimation correcte du comportement de la structure modifiée dépend directement de l'estimation des mouvements de l'interface à partir de la connaissance du mouvement des points instrumentés. Les relations (2) et (3) mettent en évidence l'importance d'une construction pertinente du modèle local et de la base de déplacements $\left[\Phi_{\mathrm{Lg}}\right]$.

\subsubsection{Modèle local}

L'objectif du modèle local est de faire le lien entre la cinématique des points instrumentés et les DDL de l'interface. La réalisation d'un modèle E.F. reprenant localement la géométrie du domaine instrumenté de la structure présente plusieurs avantages et permet de répondre aux exigences de la méthodologie telles que

- la création rapide des géométries de la structure et de la modification,

- la construction d'une base de déplacements définie sur les points de mesures et à l'interface,

- la définition des mouvements sur une interface de couplage continue (au sens E.F.),

- la « régularité » de $\left[\Phi_{\mathrm{Lg}}\right]$ par rapport aux équations de la dynamique.

Ce modèle local doit être vu comme le support géométrique de la création de $\left[\Phi_{\mathrm{Lg}}\right]$ et non comme un modèle numérique représentatif du comportement de la structure étudiée.

L'estimation du comportement couplé est fortement dépendante de l'estimation des mouvements de l'interface de la structure modifiée. Pour orienter a priori la recherche des mouvements couplés, on construit un modèle local incluant le modèle E.F. de la modification.

La figure 3 illustre l'historique de la création du modèle local depuis les mesures vibratoires sur un sousdomaine de la structure étudiée jusqu'à l'identification du problème et la proposition d'une modification. Un modèle E.F. incluant la modification et reprenant la géométrie de la structure sur le sous-domaine instrumenté est alors proposé.

\subsubsection{Construction de $\left[\Phi_{\mathrm{Lg}}\right]$}

La définition des vecteurs de $\left[\Phi_{\mathrm{Lg}}\right]$ repose sur le modèle local. Les classes de mouvements telles que les modes propres ou la déflexion statique à l'interface pour un chargement correspondant aux déplacements mesurés sont 
régulières, définies sur l'ensemble du modèle local, et permettant de reconstruire les mouvements de l'interface. Cependant, ces approches sont très liées à la géométrie du modèle local et aux caractéristiques mécaniques qui lui ont été assignées. L'existence de déformations très locales sur ces classes de déplacements peut venir perturber la construction de $\left[T_{\mathrm{It}}\right]$.

Une procédure limitant l'apparition de ces comportements locaux doit être mise en place, tout en permettant la prise en compte correcte des mesures éloignées de la zone de couplage. La solution retenue consiste à condenser statiquement le modèle local sur les DDL de l'interface et les DDL proches des points de mesure (i.e. correspondant aux colonnes non identiquement nulles de $\left[C_{\mathrm{tL}}\right]$ ). Les modes propres de ce modèle condensé sont calculés, classés par fréquences propres croissantes (minimisation de l'énergie de déformation du modèle local) puis étendus sur l'ensemble du modèle local. La géométrie de l'interface est imposée par le modèle E.F. de la modification et est indépendante du reste du modèle local. Une certaine indépendance des déformées de l'interface vis-à-vis de la modélisation complète est ainsi assurée, tout en permettant la prise en compte de l'ensemble des mesures par la condensation statique sur les DDL proches des points de mesure.

De par le principe de leur construction, les vecteurs de $\left[\Phi_{\mathrm{Lg}}\right]$ seront qualifiés de « modes d'interface ».

\subsection{Indicateur de sélection}

Pour opérer la sélection parmi les vecteurs de $\left[\Phi_{\mathrm{Lg}}\right]$, un indicateur permettant d'évaluer a priori la qualité de la reconstruction des mouvements à l'interface est nécessaire. Les critères présentés ici rendent compte de deux façons différentes d'évaluer les mouvements à l'interface pour le problème couplé. On va donc comparer les restrictions à l'interface

- des déplacements correspondant aux modes propres couplés $\left[\Phi_{\mathrm{L}}^{\text {calc }}\right]$ obtenus par le calcul, notés $\left\{q_{\mathrm{I}}^{\text {coup }}\right\}$,

- des déplacements correspondant à la déflexion statique $\left[\Phi_{\mathrm{L}}^{\exp }\right] \mathrm{du}$ modèle local ne prenant pas en compte la modification en imposant des déplacements correspondant aux modes couplés calculés rapportés aux points de mesure $\left[C_{\mathrm{tL}}\right]\left[\Phi_{\mathrm{L}}^{\text {calc }}\right]$, notés $\left\{q_{\mathrm{I}}^{\text {expand }}\right\}$.

On construit alors deux indicateurs basés sur l'énergie dissipée en faisant travailler les déplacements différentiels à l'interface du $k^{\text {ième }}$ mode propre sur le modèle de la modification condensé sur l'interface

- Pour l'énergie potentielle élastique

$$
\left(\Delta_{E_{K}}\right)_{k}=\frac{\left\|\left\{q_{\mathrm{I}}^{\text {coup }}\right\}_{k}-\left\{q_{\mathrm{I}}^{\text {expand }}\right\}_{k}\right\|_{K^{M}}^{2}}{\left\|\left\{q_{\mathrm{I}}^{\text {coup }}\right\}_{k}\right\|_{K^{M}}^{2}+\left\|\left\{q_{\mathrm{I}}^{\text {expand }}\right\}_{k}\right\|_{K^{M}}^{2}}
$$

- Pour l'énergie cinétique

$$
\left(\Delta_{E_{M}}\right)_{k}=\frac{\left\|\left\{q_{\mathrm{I}}^{\text {coup }}\right\}_{k}-\left\{q_{\mathrm{I}}^{\text {expand }}\right\}_{k}\right\|_{M^{M}}^{2}}{\left\|\left\{q_{\mathrm{I}}^{\text {coup }}\right\}_{k}\right\|_{M^{M}}^{2}+\left\|\left\{q_{\mathrm{I}}^{\text {expand }}\right\}_{k}\right\|_{M^{M}}^{2}}
$$

Pour chaque mode, les variations des indicateurs en masse et en raideur permettent d'évaluer le nombre de modes à retenir dans $\left[\Phi_{\mathrm{Lg}}\right]$ pour reconstruire l'information à l'interface. Les deux techniques d'expansion à l'origine de ces estimateurs sont complémentaires.

L'expansion statique permet d'obtenir une classe de mouvements qui, projetée sur le maillage capteur, coïncide avec les déplacements mesurés. La cohérence entre les déplacements de l'interface et les déplacements mesurés est assurée. Cependant, par construction, cette technique est sensible aux erreurs d'identification des formes modales, et la validité du résultat est liée à la pertinence du modèle retenu pour réaliser l'expansion.

L'expansion sur les modes d'interface permet de régulariser le processus. La taille de $\left[\Phi_{\mathrm{Lg}}\right]$ étant inférieure au nombre de capteurs, la projection des modes étendus sur le maillage de mesure sera moins précise que par expansion statique mais les mouvements obtenus seront plus réguliers.

Les indicateurs ainsi définis ne permettent pas d'estimer directement la qualité des résultats du calcul couplé. Même si l'enrichissement progressif de $\left[\Phi_{\mathrm{Lg}}\right]$ doit permettre de mieux représenter l'ensemble des déplacements mesurés, les deux bases de représentation des mouvements de l'interface (base complète des modes d'attaches associées aux capteurs pour l'expansion statique, base des modes d'interface pour la méthode proposée) ne sont pas définies sur les mêmes sous-espaces. Cependant, si les deux estimations du mouvement de l'interface pour les modes couplés ne concordent pas, on s'attend à ce que les résultats de l'expansion ne soient pas corrects. Le comportement prédit risque d'être sensiblement différent du comportement de la structure modifiée réelle.

Ne disposant pas pour l'instant de critères plus précis, on utilisera ces deux indicateurs pour déterminer le nombre optimal de modes d'interface à conserver dans la construction de $\left[T_{\mathrm{It}}\right]$. La détermination de l'optimum de ces indicateurs reste liée à l'interprétation de l'utilisateur, aucun critère général n'étant pour l'instant disponible.

\section{Applications}

Pour illustrer la méthodologie, deux exemples sont présentés dans cette section. Le premier est une structure académique, constituée d'une plaque rectangulaire initialement raidie sur son contour. Les résultats obtenus sont comparés aux résultats d'un essai réalisé sur une maquette de la structure. Le second est un moteur à axe vertical utilisé par EDF ayant fait l'objet d'un modèle E.F. complet et recalé. 


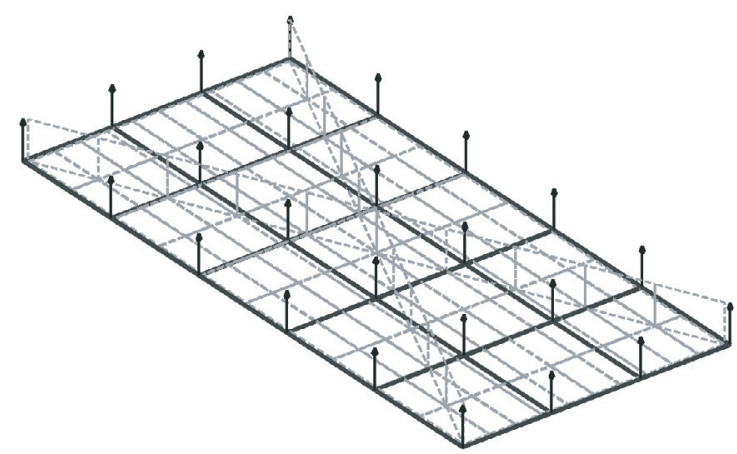

modèle local \& maillage capteur

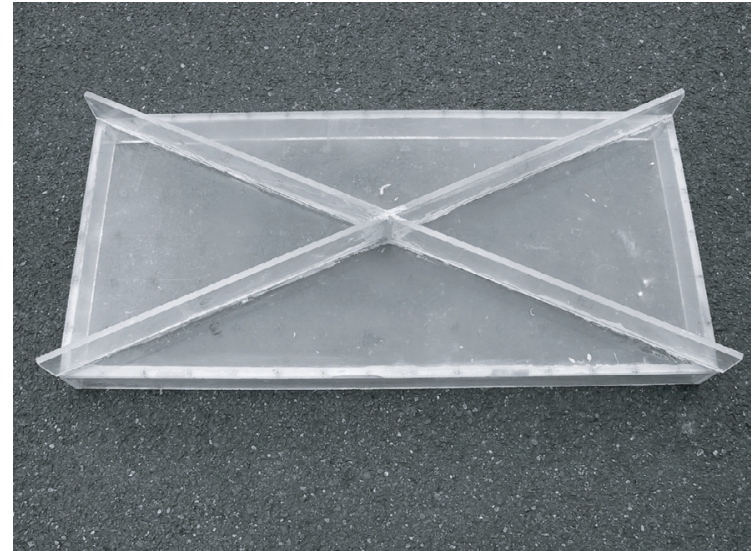

photo du démonstrateur modifié

Fig. 4. Démonstrateur académique.
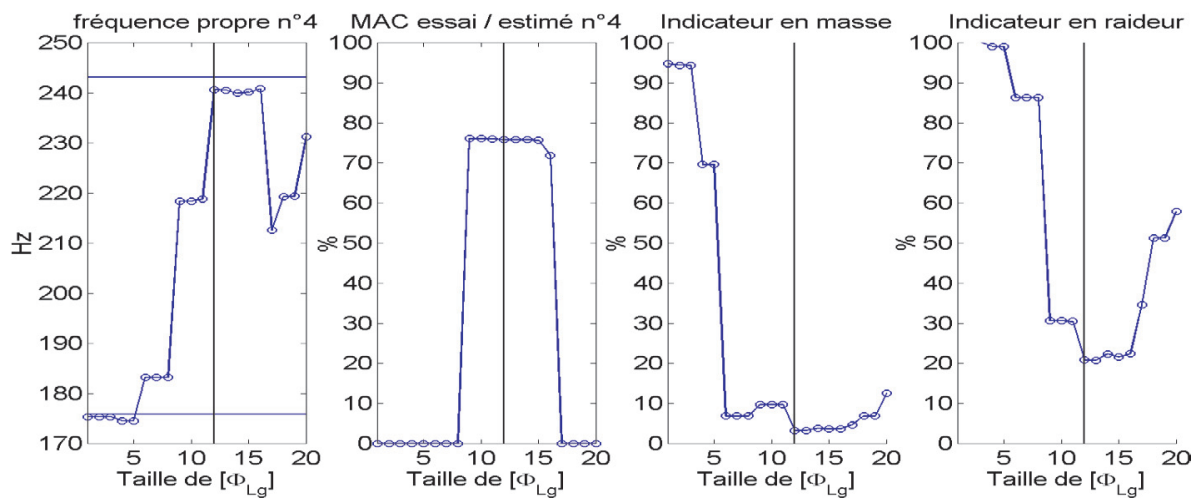

Fig. 5. Évolution de la fréquence propre estimée du mode No. 4 en fonction du nombre de modes d'interface retenu pour construire $\left[\Phi_{\mathrm{Lg}}\right]$ - Fréquences propres avant et après modification représentées par les traits horizontaux sur la partie gauche de la figure.

\subsection{Essai sur un démonstrateur académique}

La structure considérée pour la mise en place de l'ensemble de la méthodologie est présentée sur la figure 4 . Il s'agit d'une plaque plane rectangulaire initialement raidie sur son contour. La modification retenue est un croisillon rapporté sur les diagonales de la plaque. Une analyse modale est réalisée et les quatre premiers modes propres de la structure avant et après modification sont identifiés. Seuls les déplacements hors du plan du démonstrateur sont instrumentés. Les résultats de l'analyse modale et du calcul de l'effet de la modification sont présentés dans le tableau 1.

Ce cas test permet d'évaluer la méthode dans le cas d'une modification ayant un impact important sur la dynamique de la structure testée. La configuration capteur retenue pour l'analyse expérimentale est volontairement non coïncidente pour montrer l'intérêt de cette démarche vis-à-vis des méthodes classiques de modification structurale.

Les résultats présentés dans le tableau 1 sont ceux obtenus pour la taille de $\left[\Phi_{\mathrm{Lg}}\right]$ considérée comme optimum. Cet optimum est déterminé par l'étude de l'évolution des indicateurs en fonction du nombre de modes d'interface.
Tableau 1. Récapitulatif des résultats.

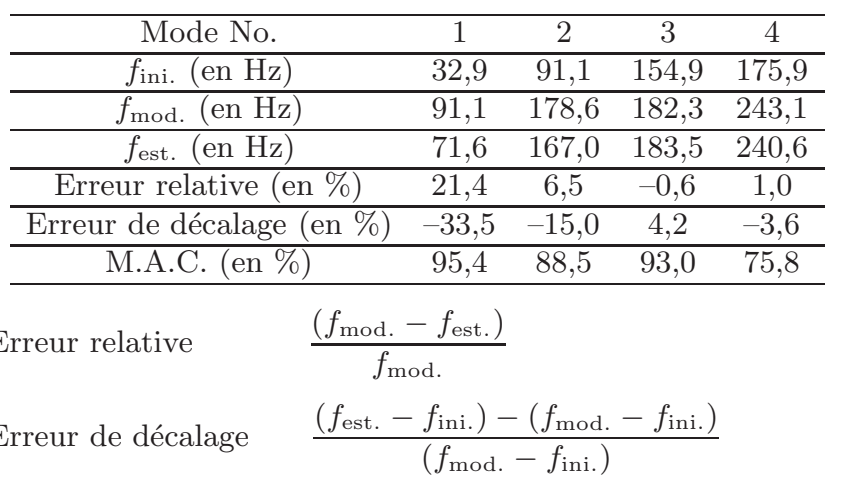

avec : $f_{\text {ini. }}$ fréquence propre avant modification, $f_{\text {mod. }}$ fréquence propre après modification, $f_{\text {est. }}$ fréquence propre estimée par la méthode proposée, M.A.C. entre les déformées identifiées et les déformées estimées (structure modifiée).

La figure 5 présente les évolutions pour le mode No. 4, parallèlement à l'évolution de la fréquence propre estimée.

La mauvaise estimation de la première fréquence propre est due à l'absence de mesure dans le plan du 

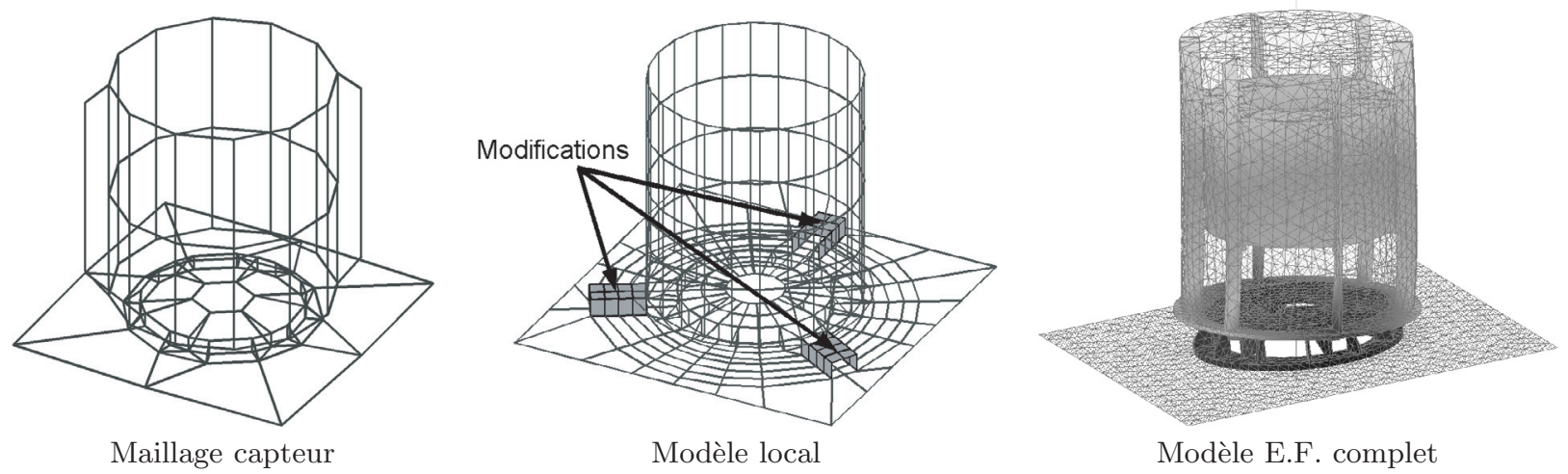

Fig. 6. Modèles du moteur de pompe.

Tableau 2. Estimation de l'effet des modifications sur les 3 premières fréquences propres du moteur.

\begin{tabular}{cccc}
\hline Mode & $\begin{array}{c}\text { Basculement du corps } \\
\text { du moteur }\end{array}$ & $\begin{array}{c}\text { Torsion autour de l'axe } \\
\text { du moteur }\end{array}$ & $\begin{array}{c}\text { Pompage du corps } \\
\text { du moteur }\end{array}$ \\
\hline$f_{\text {ini. }}($ en Hz) & 26,1 & 62,0 & 90,0 \\
$f_{\text {est. }}($ en Hz) & 45,6 & 83,2 & 91,5 \\
$f_{\text {E.F. }}($ en Hz) & 44,0 & 89,1 & 95,6 \\
\hline
\end{tabular}

$f_{\text {ini. }}$ Fréquence propre avant modification. $f_{\text {est. }}$ Fréquence propre estimée par la méthode de modification structurale proposée. $f_{\mathrm{E} . \mathrm{F}}$. Fréquence propre obtenue par calcul E.F.

démonstrateur. Cette absence est aussi pénalisante pour les méthodes classiques que pour la méthode proposée. L'étude numérique présentée dans [9] montre que, dans tous les cas, la connaissance de ces déplacements permet d'améliorer significativement la qualité des résultats.

On a montré par ailleurs dans [9] que, pour un nombre de capteurs équivalent, la méthode proposée ici donnait des résultats comparables aux méthodes de modifications structurales classiques (i.e. avec un nombre important de points de mesure sur l'interface). Ce point est capital pour cette étude, puisqu'il permet de conclure à la possibilité d'estimer l'effet d'une modification sur une structure, avec une qualité comparable aux méthodes classiques, pour un essai quelconque. Cela signifie qu'il est possible de tester l'influence de plusieurs modifications pour un même essai, mais aussi que cette étude peut se faire en une seule étape.

\subsection{Utilisation de données expérimentales sur un moteur de pompe}

Un certain nombre de moteurs à axe vertical utilisés par EDF présentent des niveaux vibratoires importants en raison de la présence d'un mode propre de basculement dont la fréquence propre est proche de la vitesse de rotation. La modification étudiée consiste en trois pièces rigides rapportées pour décaler les premières fréquences propres du moteur.

Pour évaluer l'effet de différentes modifications, un modèle E.F. (environ 150000 DDL) a été élaboré, puis ajusté à l'aide d'une analyse modale expérimentale (112 points de mesures dans les 3 directions). La mise au point du modèle a nécessité environ une année. La réalisation du modèle local et la mise en œuvre de la méthode proposée, en dehors de l'analyse modale déjà réalisée, a duré une semaine. Le maillage capteur, le modèle local et le modèle E.F. complet sont présentés sur la figure 6 .

Les résultats obtenus par les deux approches sont comparés dans le tableau 2. Ces résultats ne présument pas du comportement réel de la structure modifiée, puisque la modification n'a pas été réalisée à ce jour. Cependant, ils permettent de juger de la validité de l'approche par LMME-SDM vis-à-vis de la construction d'un modèle E.F. très complexe de la structure testée.

Le modèle local, bien que grossier, permet de bien représenter la cinematique des premiers modes propres de la structure. Les masses généralisées ont été correctement estimées pour construire un modèle dynamique du moteur pertinent. Le temps nécéssaire à la réalisation de l'ensemble de ces étapes reste sensiblement plus court que celui nécessaire à la construction d'un modèle E.F. recalé. Les gains de temps potentiels sont donc clairement illustrés, pour des résultats de qualités équivalentes.

\section{Conclusion}

Des évolutions apportées aux méthodes de modification structurale ont été présentées. La méthodologie exposée offre la possibilité d'exploiter un essai vibratoire quelconque pour étudier l'influence d'une modification sur le comportement d'une structure. En s'appuyant sur la réalisation d'un modèle local et l'expansion des données expérimentales, le couplage sur une interface non instrumentée est rendu possible. Le modèle local est avant tout un modèle géométrique devant permettre la création 
d'une base de déplacements régulière définie sur l'interface, les modes d'interface. Une base d'expansion est construite à partir d'une sélection des modes d'interface. Deux indicateurs a priori basés sur la comparaison du mouvement estimé à l'interface permettent de déterminer le nombre optimal de modes à retenir.

Deux exemples sont présentés pour illustrer quelques avantages de la méthodologie proposée. Le cas du démonstrateur académique démontre qu'il est possible d'obtenir une qualité de résultats équivalente à celle obtenue en appliquant les méthodes classiques de liaison point à point tout en s'affranchissant des contraintes sur la position des capteurs. Cet exemple montre aussi la possibilité de tester plusieurs modifications à partir d'un unique essai. Le gain de temps réalisé par rapport à la construction d'un modèle E.F. recalé est montré avec l'exemple du moteur à axe vertical. L'estimation de l'effet d'une modification est identique pour une durée de mise en œuvre d'un ordre de grandeur inférieur, dans un cas où les méthodes classiques de modification structurale ne sont pas applicables.

Ces bons résultats sont encourageants pour la suite de l'étude. Certains paramètres, tels que l'influence de la configuration du maillage de capteurs ou encore la géométrie du modèle local ont été étudiés dans [9]. D'autres sont encore à étudier pour orienter la construction du modèle local et l'optimisation de l'analyse modale, comme l'influence des caractéristiques mécaniques du modèle local ou la propagation des erreurs d'identification dans le processus (en particulier les erreurs sur les masses généralisées). La maîtrise de ces paramètres doit permettre une meilleure interprétation des résultats.
La maîtrise de ces paramètres pourrait éventuellement conduire à la construction d'un indicateur de confiance dans l'estimation du comportement de la structure modifiée.

\section{Références}

[1] N. Maia, J. Silva, et al., Theoretical and experimental modal analysis. Research Studies Press, first edition, 1997

[2] W. D'Ambrogio, A. Sestieri, Using distributed modifications to change the dynamic behaviour of structures, IMAC XVII (1999) 763-768

[3] W. D'Ambrogio, A. Sestieri, Coupling theoretical data and translational frf to perform distributed structural modification, Mechanical Systems and Signal Processing 15 (2001) 157-172

[4] K.B. Elliot, L.D. Mitchell, Structural modification using beam elements, IMAC V 2 (1987) 956-965

[5] B.J. Schwarz, M.H. Richardson, Structural modifications using higher order elements, IMAC XV (1997) 313-318

[6] E. Balmès, Méthodes de modification structurale, application à la tkj de Chooz b2, Rapport contrat EDF/DER P51/C28961/EP957, 2000

[7] G.T. Herman, Image Reconstruction from Projections, Academic Press, first edition, 1980

[8] E. Balmès, Sensors, degrees of freedom, and generalized modeshape expansion methods, IMAC XVII (1999)

[9] M. Corus, E. Balmès, L. Billet, Amélioration de méthodes de modification structurale par utilisation de techniques d'expansion et de réduction d'interface, Rapport EDF HT61/01/022, 2001 